\title{
Molecular evidence for a new dispersal route of inoculum source for Puccinia striiformis f. sp. tritici in central and eastern China
}

\author{
liang huang ${ }^{1}$, Heng Yang ${ }^{2}$, Chongjing Xia², Aolin Wang ${ }^{2}, \mathrm{Li} \mathrm{Gao}^{2}$, Bo Liu², Yilin Zhou ${ }^{2}$, \\ Xiaohui Kang ${ }^{3}$, Min Zhang ${ }^{4}$, Wanquan $\mathrm{Chen}^{2}$, and Taiguo $\mathrm{Liu}^{2}$ \\ ${ }^{1}$ Affiliation not available \\ ${ }^{2}$ Chinese Academy of Agricultural Sciences Institute of Plant Protection \\ ${ }^{3}$ Southwest University of Science and Technology \\ ${ }^{4}$ Sichuan Agricultural University - Chengdu Campus
}

November 6, 2020

\begin{abstract}
Wheat stripe rust, caused by Puccinia striiformis f. sp. tritici (Pst), is one of the most destructive diseases of wheat in China. Due to the pandemic in $2017^{\sim} 2018$, a total of 709 single-lesion samples, including 285 collected in 7 provinces in 2017 and 424 collected in 4 provinces in 2018, were isolated to explore the underlying causes. The population structure was analyzed by using 13 simple sequence repeat (SSR) makers. 306 multi-locus genotypes (MLGs) were detected and the genetic diversity was different among populations. Regardless of the year difference, 11 populations were clustered into two group: Gansu, Sichuan and Shaanxi belong to one group, while Yunnan, Guizhou, Hubei, and Anhui belong to another group. Genetic identity and shared genotypes along with the minimum spanning network (MSN) showed the Pst population from Sichuan play an intermediate role of genetic communication between Yunnan-Guizhou and Gansu. Since Berberis spp. has been identified as the alternate host of Pst, our results also provided a molecular evidence of potential sexual reproduction in Gansu population. In addition, the closer relationship between Yunnan-Guizhou and Hubei-Anhui was demonstrated. It was showed the role of Pst population in Yunnan-Guizhou might was underestimated in epidemiology. Previously, it was believed that the inoculum was limited locally. Our study provided strong evidence that the Pst inoculum source of Yunnan-Guizhou dispersed to Hubei areas by the upper airflow, and migrated to Anhui finally detriment Huang-huai regions.
\end{abstract}

\section{Introduction}

As the third largest cereal staple in China, the sustained development of wheat has made great important contributions to ensuring domestic food security (He et al. 2018). Wheat is growing almost in all provinces in China, Henan, Shandong, Hebei, Jiangsu, and Anhui are the 5 largest wheat-producing provinces, supporting more than $60 \%$ of the national production (Wan et al. 2007). Meanwhile it is threatened by biotic and abiotic stresses constantly every year. Stripe (yellow) rust, caused by Puccinia striiformis f. sp. tritici (Pst), is an important fungal disease. With the long-distance dispersal of Pstpathogen, it could cause infections more than thousands of kilometers away from initial infected areas which makes it one of the most destructive diseases of wheat in many regions of the world, especially in China (Zeng and Luo 2006, Chen et al. 2014). China has the largest independent epidemic region with more than 20 million hectares of wheat affected by stripe rust annually (Stubbs 1988). The most destructive epidemics occurred in 1950, 1964, 1990, 2002, 2009 , and 2017, which caused yield losses exceeded 6.0,3.2, 1.8, 1.3 and 1.5 million metric tons, respectively (Huang et al. 2020). Growing disease-resistance varieties have been considered to be the most economical, effective and environmental friendly measures to control wheat stripe rust (Chen et al. 2009). However, due to the rapid genetic variations, the disease-resistant varieties might lose resistance in a few years after their release. The mechanisms of the variability are considered as mutation, parasexuality, selection and somatic 
recombination (Mboup et al. 2009, Duan et al. 2010, Hovmøller et al. 2011), sinceBerberis spp. were found as alternate hosts for the wheat stripe rust pathogen (Jin et al. 2010), the sexual recombination could become a new research hotspot (Wang et al. 2016, Tian et al. 2016). Based on this situation, wheat resistance research alone is not enough to completely prevent the outbreak of wheat stripe rust in production.Pst is transmitted by airflow and then causes disease epidemic, its epidemic varies from year to year due to different climatic conditions, therefore, it is particularly important to understand the genetic structure of the pathogen population along with the epidemic pattern continuously and thoroughly for the prevention and management of wheat stripe rust.

Traditional studies on population structure of Pst mainly determined by races surveys (Chen et al. 2010, Chen et al. 2009). With the development of molecular makers, various types of makers, such as random amplified polymorphic DNA (RAPD), restriction fragment length polymorphism (RFLP), amplified fragment length polymorphism (AFLP), were applied to analyze the population structure of Pst . However, these maker systems detected only low levels of intraspecific polymorphisms (Song et al. 2005). Microsatellite DNA markers (Simple sequence repeat, SSR), as one of the most informative genetic makers, were widely used to estimate the population structure of Pst and other pathogen all over the world (Bahri et al. 2011, Brar et al. 2018, Enjalbert et al. 2005, Holtz et al. 2014, Zhan et al. 2016).

Intensive research of wheat stripe rust in China, involved large-scale investigation and fixed-point monitoring, combined with geographic, climatic, races and molecular marker techniques and so on, have been divided into three epidemiological regions: oversummering regions (Autumn sources of Pst inoculum), overwintering regions (Spring sources of Pst inoculum), Spring epidemic areas, which constituted an independent and complete wheat stripe rust circulatory system in China (Chen et al. 2013). The pathogen can oversummer and overwinter in different specific regions and then cause the spring epidemic. The southern Gansu and northwest Sichuan have been considered as the most important oversummering areas and the origin of new races of wheat stripe rust in China. Meanwhile, the pathogen can oversummer in Yunnan-Guizhou regions. However, previous studies suggested that the role of oversummering inoculum in these areas was limited locally (Chen et al. 2013). In addition, the relationship of Pst between Yunnan-Guizhou and other regions of China is unclear entirely.

It is by now generally accepted that the northwest winter regions are the most important over-summering area of Pst and the major source of inoculum for wheat infection in autumn of china (Chen et al. 2014). The northwest over-summering pathogen urediniospores dispersal to Chengdu Plain and Jianghan River Basin in autumn annually, then increasing in this region at winter and the major spring inoculum source of stripe rust disease. However, according to the field survey in 2017, the incidence area of autumn wheat seedlings in Northwest wheat areas such as Gansu was the smallest in the past 10 years, but the wheat stipe rust was more severe in southwest areas (Sichuan, Yunnan, Guizhou) (Huang et al. 2018a, Huang et al. 2018b). This led us to speculate the inoculum sources responsible for the wheat stripe rust epidemic in Hubei-Anhui in 2017 might be from Southwest instead of Northwest of China. The main objectives of the present study were to: (1) explore the possibility that the pathogen of wheat stripe rust in the eastern wheat areas originated from Yunnan-Guizhou region; (2) the relationship of the pathogen between Yunnan-Guizhou and Gansu region; (3) the reproduction modes of $P s t$ in different regions.

\section{Materials and methods}

\section{Sample collection and DNA preparation}

A total of 709 samples from 7 different provinces in China were collected in 2017 and 2018(some of the samples were collected by the research group and cooperative researchers and some from the trap nursery fields settled at 30 locations in China). In the targeting province, samples were collected from no less than three fields and the fields are far from each other. Samples taken from the same year and province were considered as one population, giving 11 populations in total (Table S1). Leaves with fresh single-lesion of stripe rust and no symbols of other pathogens were sampled and each leaf was encapsulated in an envelope to avoid cross-contamination. 
For DNA extraction according to a modified CTAB method, the procedure was as follows: single-lesion was cut from collected samples and each of them were transferred to a $2-\mathrm{mL}$ centrifuge tube with $0.4 \mathrm{~g}(\Phi=1 \mathrm{~mm})$ and $0.2 \mathrm{~g}(\Phi=0.1 \mathrm{~mm})$ glass beads, and then ground by a FastPrep 24 shaker (MP Biomedicals, USA) for 1.5 min at the speed of $6.5 \mathrm{~m} \mathrm{~s}^{-1}$. The $660 \mathrm{uL} 1.1 \%$ CTAB $(\mathrm{pH}=8.0)$, and $10 \mu \mathrm{L}$ of $20 \mathrm{mg} \mathrm{mL}^{-1}$ proteinase $\mathrm{K}$ was added, mixed gently, and incubated in water bath at $65^{\circ} \mathrm{C}$ for $1 \mathrm{~h}$ (the tubes were gently mixed every 15 $\min$ ). The $660 \mu \mathrm{L}$ Phenols/chloroform/isoamyl (25:24:1) was added, the mixture was inverted until totally blending and centrifuged by a high speed refrigerated centrifuge (Eppendorf, Germany) at $12000 \mathrm{r} \mathrm{min}^{-1}$ for $10 \mathrm{~min}$ at $4^{\circ} \mathrm{C}$. The aqueous phase was shifted to a new tube, equal volume chloroform/isoamyl alcohol (24:1) was added and extracted. The supernatant was transferred to $1.5-\mathrm{mL}$ tube, $5 \mu \mathrm{L} 100 \mu \mathrm{g} / \mathrm{mL}$ RNA enzyme was added and put into water at $37^{\circ} \mathrm{C}$ for $1 \mathrm{~h}$, and then extracted twice with the same volume of chloroform/isoamyl (24:1). Three fifths volume of isopropanol which was cooled in advance was added and kept in $-20^{\circ} \mathrm{C}$ overnight. Subsequently, centrifugation for 10 minutes at $4^{\circ} \mathrm{C}$ and $12000 \mathrm{r} \mathrm{min}^{-1}$. Discard the supernatant and rinsed the sediment twice with $1000 \mu \mathrm{L}$ of pre-cooled $70 \%$ ethanol. Dissolved the DNA with $30 \mu \mathrm{L}$ dd $\mathrm{H}_{2} \mathrm{O}$ at $4^{\circ} \mathrm{C}$ after drying, the concentration of the DNA was measured by Nano Drop (ND-1000, USA) and the DNA samples were diluted to $50 \mathrm{ng} / \mu \mathrm{L}$ and stored at -20 until further use.

\section{SSR Genotyping}

Initially, 32 primers were selected for the preliminary experiment, of which 13 primers with polymorphisms were used for the subsequent experiment (Table 1). The PCR reaction was carried out in a total volume of $25 \mu \mathrm{L}$ including $1 \mu \mathrm{L}$ of prepared DNA, $12.5 \mu \mathrm{L}$ of $2 \times$ Unique $^{\mathrm{TM}}$ Taq Super Master Mix (Novogene, Beijing, China), $9.5 \mu \mathrm{L}$ of sterile $\mathrm{ddH}_{2} \mathrm{O}$ and $1 \mu \mathrm{L}$ of each forward and reverse primer respectively, the conditions were as following: initial denaturation at 94 for $5 \mathrm{~min}, 35$ cycles at 94 for 30s, 56 for 30s (according to the annealing temperature of the primers) and 72 for $45 \mathrm{~s}$, with a final extension at 72 for $10 \mathrm{~min}$.

The amplification products were diluted at the ratio of 1:50 after analyzed on an $2 \%$ agarose gel, and then $1 \mu \mathrm{L}$ diluted product was added into the mixture of $9 \mu \mathrm{L}$ Hidi (Hi-Di Formamide for 3500 Dx/3500xl Dx)+Liz500 (GeneScan 500 LIZ Size Standard), the ratio of Hidi to Liz500 is 1000:15. Amplicons length of each SSR were determined using an ABI 3500xl Genetic Analyzer (Applied Biosystems, USA). The length of the fragments was scored by using GeneMaker v2.7.0 (SoftGenetics, USA).

\section{Data Analysis}

A data set of the samples was conducted in Microsoft Excel following the GenALEx 6 data format (Peakall and Smouse 2012), which contain all the allelic size of each locus. The $\mathrm{H}_{\mathrm{o}}$ (observed heterozygosity) (Charlesworth 1998) (Meirmans and Hedrick 2011) and $\mathrm{H}_{\mathrm{e}}$ (expected heterozygosity) (Charlesworth 1998) were also calculated through GenALEx to evaluated the usefulness of the primers (Table 2).

POPPR (version 2.5.0) (Kamvar et al. 2014), an package of $\mathrm{R}$ was used to explore the following analysis: To assess how much power the microsatellites have to discriminate between distinct individuals, the phylogenetically uninformative loci analysis (the default is $(2 / \mathrm{N})$, where $\mathrm{N}$ equals the number of individuals in the data set) and a genotype accumulation curve (Grünwald et al. 2017) was plotted. The POPPR function info table was used to discard the locus with missing data exceed 10\%. Genetic diversity was assessed by the following analysis: (1) The diversity of alleles at each locus by calculating Simpson's index (1-D); (2) The evenness of the alleles at each locus (Evenness); (3) Number of the multilocus genotypes (MLGs) observed; (4) Genotypic richness is measured by the number of expected MLG (eMLG), which is an approximation of the number of genotypes that would be expected at the largest shared sample size based on rarefaction and the shared sample size should [?] 10; (5) Corrected Simpson's Index (Corrected lambda) was used to avoid the influence by population size (Simpson 1972), (6) Nei's unbiased gene diversity (Hexp) (Nei 1978); (7) Genotypic evenness (E.5) (Grunwald et al. 2003). The following analysis is based on data from cloned populations: The standardized index of association (rbarD) were used to determine the mode of the reproduction (Brown et al. 1980, Goss et al. 2014, Kamvar et al. 2014). The Analysis of Molecular Variance(AMOVA) was performed to detect the variations and differentiation in the given population (Excoffier et al. 1992), including four level: variations between year, variations within samples, variations between samples within 
province and variations among provinces. The dendrogram based on Provesti's distance, Minimum spanning networks (MSN) based on Bruvo's distance (Bruvo et al. 2004) together with genetic identity and genetic distance based on Nei's Original Measures (Nei 1972) through Popgene (Version 1.32) were carried out to investigate the relationship between the different populations.

\section{Air mass transport with HYSPLIT}

There are no local sources of Pst in Hubei as described Wan et al (Wan et al. 2007), since the pathogens cannot complete its oversummer or overwinter in this region. Two representative locations, including Jingmen and Xiangyang of Hubei were selected according to the field surveys and based on model calculations with the HYSPLIT model by back tracking at a height of $2000 \mathrm{~m}$ and $3000 \mathrm{~m}$ above ground. Further analysis of the air mass transport was performed using GIS techniques (MeteoInfoMap v. 2.2.1, China).

Monthly meteorological data (i.e., NCEP/NCAR 2.5degx2.5deg Reanalysis Data) were obtained from the National Centers for Environmental Prediction and the National Center for Atmospheric Research using the HYSPLIT model for the period 1948 to present. Data were outputted four times a day (i.e., at 00, 06, 12, and 18 UTC) and data contain variables such as the $\mathrm{u}$ - and v-wind components, temperature, and humidity. The HYSPLIT model is used to calculate trajectories, which are loaded into the TrajStat (the geographic information system based software) as an external process. Thereafter, the trajectory data of two months were combined and the clustering function is used for dimensionality reduction.

The starting date of HYSPLIT backward trajectory model was determined by the onset date which the seedling disease of stripe rust in Hubei. For each year, the model's running period for the selected locations was from 1 November to 31 December. In each simulation, the pathogen spores were released four times a day (i.e., at 00, 06, 12, and 18 UTC) and the total run time for each simulation was $36 \mathrm{~h}$. In this study, the possibility of the potential inoculation source being transferred to Hubei province was analyzed.

\section{RESULTS:}

\section{Basic statistics and loci information}

A total of 306 MLGs were detected from the 709 isolates. Seventy-nine MLGs were re-sampled and 10 MLGs were re-sampled more than ten times, the predominant genotype MLG-165, MLG-163 and MLG-61 were detected in more than 2 provinces and re-sampled more than 30 times (78, 56 and 33 times respectively) (Table S2) .

All 13 SSR markers used in this study were phylogenetically informative, polymorphic, and the number of alleles at different loci ranged from 2 to 8 , resulting in 49 alleles in total. The observed heterozygosity $\left(\mathrm{H}_{\mathrm{o}}\right)$ and expected heterozygosity $\left(\mathrm{H}_{\mathrm{e}}\right)$ were similar for four of the thirteen loci, five of the other 9 loci have higher $\mathrm{H}_{\mathrm{o}}$ and the remaining four have lower. The values of 1-D and Hexp of the loci are similar and ranged from 0.033 to 0.683 and 0.033 to 0.684 , respectively (Table 1 ).

\section{The genetic diversity of different population}

Among the seven populations in 2017, Gansu population had the highest eMLG value, and the value of Shaanxi population was close to Gansu. The Yunnan, Guizhou, Sichuan populations had similar eMLG values $(9.11,8.37,9.53$, respectively), Hubei population had the lowest eMLG values. In 2018, the eMLG values of Gansu and Sichuan populations were similar. Hubei population had the lowest eMLG in 2018, consistent with the results of 2017, indicating the genotype richness of here is low. The values of E.5 were measured to represent the evenness of population genotypes, which were ranged from 0.433 (2018_HB) to 1 (2017_GS), a high value demonstrates a higher genotype evenness. The genotypic and gene diversity of different populations were vary from each other, 2017_GS population had the highest corrected lambda and Hexp of the seven population analyzed in 2017, 2017_YN and 2017_GZ had similar corrected lambda as well as Hexp. The value of corrected lambda was lowest in 2018_HB population, however, 2018_YN population had the lowest Hexp value, 2018_SC population had higher genetic diversity than 2018_GS population, meanwhile, the sample size of 2018_SC is much more than 2018_GS population (Table 2). 


\section{Test for linkage disequilibrium}

The index of association ( $\mathrm{rbarD}$ ) were used to test if populations are in linkage equilibrium or not, the rbarD are expected to be zero if populations are sexuality and greater than zero if there is clonality. Here we found significant support to reject the hypothesis that alleles are equilibrium across loci with $\mathrm{P}<0.001$, indicating that random mating or sexual reproduction is not taking place within the majority of the populations, however, 2018_GS population had the lowest $\operatorname{rbarD}(\operatorname{rbarD}=0.029, \mathrm{P}=0.036)$ which given a clue of the possibility of sexual reproduction of this population (Fig 1).

\section{Population differentiation and relationship between different pairs}

The Analysis of Molecular Variance (AMOVA) showed almost no variations $(<0.01 \%, \mathrm{P}=0.418)$ were present between the population of 2017 and 2018, indicating the stripe rust pathogens populations remained stable between these years, the majority of the variations were present within samples $(70.27 \%, \mathrm{P}=0.001)$, it was worth to note that $8.11 \%$ variations present between populations within a year which showed significant differences between populations ( $\mathrm{P}=0.001$ ) (Table 3 ). Nei's original measures of genetic identity and genetic distance indicated that in 2017, the population of Yunnan had the highest genetic identity comparing with Guizhou, Hubei and Anhui populations had highest genetic identity with Yunnan and Guizhou populations. In 2018, Gansu population had the highest genetic identity with Sichuan population, as well as between Yunnan and Hubei, other combinations were lower (Table 4). The dendrogram according to Provesti's distance was constructed (Fig 2), on the difference of 0.08, the eleven populations were divided into two groups. Populations of Gansu and Sichuan were always divided into same group regardless of the year, the same situation for populations of Yunnan and Hubei. 2017_SX population was clustered into group 1, 2017_GZ and 2017_AH population were clustered into group 2. In addition, among the detected 306 MLGs, 32 were shared in different pair combinations. Shared MLGs are frequently found in Yunnan, Guizhou, Hubei and Anhui, indicating a closer relationship between these population. Meanwhile, a part of shared MLGs were also found in Yunnan, Guizhou and Sichuan, five of six shared MLGs in Gansu were detected in Sichuan, lower numbers were shared between other pairs (Table S2).

Since we found the populations of Hubei and Anhui had closer relationship to Yunnan and Guizhou than that to Gansu, an MSN was built to show the relationship between these populations (Fig 3). Here we found that the populations of 2017_YN and 2017_GZ in the tree heavily contributed to the major groups of MLGs found in the population of 2017_HB and 2017_AH, which was consistent with the results of 2018, the relationship between Yunnan and Hubei was closer than between Gansu and Hubei. Another MSN was conducted to explore the relationship between Yunnan-Guizhou and Gansu (Fig 4). No matter which year, the population relationship was closer between Sichuan and Yunnan-Guizhou or between Sichuan and Gansu, but there was still some relationship between Yunnan-Guizhou and Gansu. What is more significant is that individuals of Sichuan population existed in places where Yunnan-Guizhou and Gansu are linked.

So far, all the evidence points out that i) the population of Hubei-Anhui is more close to that of YunnanGuizhou; and 2)stripe rust pathogens of Sichuan population play an intermediate role of genetic communication between Yunnan-Guizhou and Gansu populations.

\section{Backward trajectory analysis}

It is generally known that Wheat stripe rust spread over a long distance by airflow. In order to understand the origin of the Hubei pathogens, we set up a HYSPLIT backward trajectories model which was from locations in Hubei province (Jingmen and Xiangyang), in November to December at 2016 and 2017. The result showed the airflow mainly come from southwest and northwest of China (Fig 5 and Fig 6). Once the southwest warm moist air meets the northwest strong cold air in Hubei area, rainfall will be formed, meanwhile the spores of Pstcould land on susceptible hosts.

\section{Discussion}

\section{Genetic diversity was clearly demonstrated by mutilocus analysis}


Many studies focus on Gansu and northwest Sichuan in China (Duan et al. 2010, Liang et al. 2013, Liang et al. 2016, Zhan et al. 2013), and lack of understanding of other areas. The genetic diversities varying from region to region were found in different provinces of China. Mutation as the ultimate source resulting in new allele and genotypes and exist in completely all individuals regardless of the reproduction method, whereas somatic recombination, parasexual and sexual reproduction are only impacting genotype diversity (Hovmoller et al. 2011). Host selection tends to favor the most fit individuals that would influence the frequencies of genotypes. Zhan (Zhan et al. 2013) reported 29.5\% variations among varieties, furthermore, the varieties difference only explained $2 \%$ genetic variation according to Berlin (Berlin et al. 2013). Two years eMLG values, E.5 values and corrected Simpon's index given us adequate evidence that Gansu province was the diversity center of Pst in China. Hubei and Anhui were divided into spring sources of inoculum and spring epidemic areas respectively (Chen et al. 2013) which indicated that the initial inoculum of these areas were coming from other regions. If the disease occurred by exotic inoculum source of Pst, the earlier the pathogen comes, the more cloned the population will be. For instance, the lower genotypic diversity was found in Hubei compared to Anhui in 2017. At the same time, Hubei-Anhui population had the lowest genetic diversity compared with the other populations.

\section{Linkage disequilibrium analysis showed potential sexual reproduction in Gansu population}

The reproductive mode of Puccinia striiformis f. sp.tritici has long been considered as asexual, populations in most areas of the world (Brar et al. 2018, Chen et al. 2002, Enjalbert et al. 2005, Holtz et al. 2014) consistent with this hypothesis which have low genetic diversity, meanwhile, populations with high diversity were found in Gansu province (Liang et al. 2016, Wan et al. 2015), China, and Pakistan (Bahri et al. 2011). In our study, plenty of MLGs were re-sampled in different locations, the presence of a particular MLG in great excess is often the most robust and significant evidence of clonal reproduction (Tibayrenc et al. 1991). The value of rbarD were $0.086(\mathrm{P}<0.001)$, it's more like a cloned population if we did not differentiate the populations by regions and years. However, the characteristic of Gansu population in 2018 was significantly different from those of other populations by the value of $\operatorname{rbarD}$ was $0.029(\mathrm{P}=0.036)$, and we identified the highest genotypic richness, evenness and corrected Simpson's diversity index of Gansu population in 2017, but the rbarD was higher than that in 2018, this was maybe due to the low sample size. Geographical isolation and local adaptation will affect the genetic evolution of the Pst (Chen et al. 2014). The feature of other populations showed the same situation with high rbarD value with $\mathrm{P}<0.001$ as well as the lower genotypic diversity. Sexual reproduction is advantageous, because it can combine various beneficial factors through recombination, which facilitates adaption (Luijckx et al. 2017). Since Berberis spp. have been identified as the alternate host for the completion of the sexual life cycle of P. striiformis (Jin et al. 2010), and kinds of Berberis spp.are widely distributed in Gansu province, which can contribute to the occurrence of stripe rust to adjacent wheat (Wang et al. 2016, Zhao et al. 2018). Our results provided a molecular evidence of potential sexual reproduction in Gansu population. However, how sexual reproduction affects disease epidemics, especially to what extent, is still unknown and an urgent problem to be investigated.

Minimum spanning network showed Sichuan population play an intermediate role of genetic communication between Yunnan-Guizhou and Gansu

Yunnan, Guizhou and Sichuan are adjacent to each other, the genetic identity between Sichuan and YunnanGuizhou was high and a certain number of shared genotypes were found in these regions gave the evidence for strong interprovince exchange, this was consistent with the results of Liu (Liu et al. 2016), meanwhile, same situation was found between Sichuan and Gansu (Liang et al. 2016), our results also suggested strong genetic identity between Gansu and Sichuan. Although the genetic identity between Yunnan-Guizhou and Gansu was the lowest, there is still correlation between these two regions. MSN showed that partial individuals of Yunnan-Guizhou were close to those in Gansu, meanwhile, Sichuan population heavily contributed to the relationship between Yunnan-Guizhou and Gansu, since Sichuan areas existed between Yunnan-Guizhou and Gansu regions. Analysis of meteorological data from 1997-2006 showed, the sources of inoculum in Sichuan, Yunnan, and Guizhou have a high probability of impacting the disease epidemics in northern and northwestern China at autumn (Wang et al. 2010). 


\section{Population genetic analysis showed closer relationship between Yunnan-Guizhou and Hubei-}

Anhui

The AMOVA showed most of the genetic variations existed within samples, which were consistent with the characteristics of high variations of $P$ st genome, randomization test showed significant differences presented in all levels except for the samples variations between year, this was in accord with the results of Berlin et al. (Berlin et al. 2013), who examined the populations of Puccinia graminis f. sp.avenae (Pga) and showed only a small extent genetic variations presented in year of collection. Differences in geospatial distribution often lead to population differentiation (Schaal 1980). Yunnan and Guizhou are located in Yunnan-Guizhou Plateau and belong to the southwest epidemic area of wheat stripe rust in China (Zeng and Luo 2006), which have similar geographic and ecological environment, and our results showed the pairwise genetic identity was highest between Yunnan and Guizhou (0.995). The genetic identity was lower between Yunnan-Guizhou and Gansu, which may result from the geographical barrier. The genetic identity between Yunnan-Guizhou and Hubei-Anhui was similar, and shared genotypes found in these areas were much more than the other regions, meanwhile, the genetic distance calculated by Provesti's distance divided the 11 populations into two clades. Shared genotype directly reflects whether there is source exchange among different populations, but the genetic distance between other non-shared genotypes is unknown. Minimum spanning network calculated by Bruvo's distance is based on a stepwise mutation model and can handle missing data (Bruvo et al. 2004). Whether in 2017 or 2018, the population of Yunnan-Guizhou in the tree heavily contributed to the major groups of MLGs found in the population of Hubei-Anhui. With the rising global temperature, pathogenic fungi are constantly adapting to higher temperatures, moreover, with the decrease of altitude and latitude, as well as the passage of years, the high temperature tolerance of stripe rust population has been strengthened, and temperature-adaptive strains not only show higher adaptability to temperature, but also have higher virulence (Milus et al. 2009, Zhang et al. 2013). Yunnan-Guizhou region is in the south of China with low latitude, high annual temperature, and most wheat planting resistance cultivars all year round, which creates favorable conditions for wheat stripe rust pathogen to adapt to adversity stress. According to the survey, the incidence area and range of 2017 autumn seedlings infected by Pst in Northwest China were the smallest in last 10 years, whereas the occurrence was more serious in Southwest China (Huang et al. 2018a). Thus, we believed that the source of inoculum from Yunnan-Guizhou was the main cause of the epidemic of stripe rust in 2017.

\section{Air trajectory indicatedthe inoculum from Yunnan-Guizhou area might be a new sources of wheat stripe rust disease in central and east China}

Long-distance transport is an important pathogen dispersal mode, in particular, wheat stripe rust, which can be transmitted by high altitude and long distance, is the representative model, the pathogens could spread from west oversummering regions to east wheat belts of China in the $3 \mathrm{~km}$ of the upper air (Xie et al. 1993). Early studies suggested Gansu-Shaanxi and northwestern of Sichuan is the major inoculum sources of wheat autumn seedling in eastern China, and the role of Yunnan-Guizhou inoculum may be limited to local area (Chen et al. 2013). However, the results of backward trajectory analysis indicated the $3 \mathrm{~km}$ upper airflow can act as the medium of the inoculum from Yunnan-Guizhou to Hubei at autumn, along with our molecular evidence. Analysis of the spatiotemporal patterns in 2017 showed that, stripe rust was first found in 2016 December in the northwest Hubei and southern Henan regions, but the both eastern Sichuan and Chongqing were not found, and the disease was first found in 2017 March in Anhui regions (Huang et al. 2018a). Thus, we consider that the Yunnan-Guizhou sources of inoculum might transported to Hubei by upper airflow in autumn, and dispersed to Anhui in spring. So, we strongly believed that the inoculum from Yunnan-Guizhou area might be a new sources of wheat stripe rust disease in central and east China.

\section{Conclusion}

The genetic diversity of wheat stripe rust was varying from different populations. The Gansu population had the highest genetic diversity, which was consistent with many documentations. In contrast, the Hubei-Anhui populations had the lowest. Linkage disequilibrium analysis showed potential sexual reproduction in Gansu population, however, the contribution of sexual reproduction to disease epidemic deserves further study. 
Pairwise population genetic identity and other evidence showed closer relationship between Yunnan-Guizhou and Hubei-Anhui. And Sichuan population may play an intermediate role of genetic communication between Yunnan-Guizhou and Gansu. According to our study, it is important to pay attention to the inoculum source of Yunnan-Guizhou when developing disease control strategies, since Yunnan-Guizhou sources of inoculum could dispreading to Hubei through upper air in autumn, and impacted wheat belts of east China in spring.

\section{Acknowledgements}

This study was supported by the National Natural Science Foundation of China (Project 31871906, CHINASTINT project 31611130039), National Key Research and Development Program (2018YFD0200500 \& 2016YFD0300700), China Agriculture Research System (CARS-3), Agricultural Science and Technology Innovation Program (CAAS-ASTIP) and Epidemic Detection and Control of Crop Diseases and Insect Pests (2130108).

\section{Reference}

Bahri B., Shahc S.J.A., Hussaind S., Lecontea M., Enjalbertae J. and Vallavieille-Popea C.d. 2011. Genetic diversity of the wheat yellow rust population in Pakistan and its relationship with host resistance. Plant Pathology. 60(4):649-660.

Berlin A., Samils B., Djurle A., Wirsen H., Szabo L. and Yuen J. 2013. Disease development and genotypic diversity of Puccinia graminisf. sp. avenae in Swedish oat fields. Plant Pathology. 62(1):32-40.

Brar G.S., Ali S., Qutob D., Ambrose S., Lou K., Maclachlan R., Pozniak C.J., Fu Y.-B., Sharpe A.G. and Kutcher H.R. 2018. Genome re-sequencing and simple sequence repeat markers reveal the existence of divergent lineages in the Canadian Puccinia striiformis f. sp.tritici population with extensive DNA methylation. Environmental Microbiology. 20(4):1498-1515.

Brown A.H., Feldman M.W. and Nevo E. 1980. Multilocus structure of natural populations of Hordeum spontaneum. Genetics. 96(2):523-536.

Bruvo R., Michiels N.K., D'Souza T.G. and Schulenburg H. 2004. A simple method for the calculation of microsatellite genotype distances irrespective of ploidy level. Molecular Ecology. 13(7):2101-2106.

Charlesworth B. 1998. Principles of population genetics. 3rd edition (1993-re-issued 1997). By Daniel L. Hartl and Andrew G. Clark. Sunderland, MA: Sinauer 519 pp. Bioessays. 20(12):1055.

Chen W., Kang Z., Ma Z., Xu S., Jin S. and Jiang Y. 2013. Integrated management of wheat stripe rust caused by Puccinia striiformis f. sp. tritici in China. Scientia Agricultura Sinica (in Chinese). 46(20):42544262 .

Chen W., Wellings C., Chen X., Kang Z. and Liu T. 2014. Wheat stripe (yellow) rust caused by Puccinia striiformis f. sp.tritici. Molecular Plant Pathology. 15(5):433-446.

Chen W., Wu L., Liu T., Xu S., Jin S., Peng Y. and Wang B. 2009. Race dynamics, diversity, and virulence evolution in Puccinia striiformis f. sp tritici, the causal agent of wheat stripe rust in China from 2003 to 2007. Plant Disease. 93(11):1093-1101.

Chen X., Moore M., Milus E.A., Long D.L., Line R.F., Marshall D. and Jackson L. 2002. Wheat stripe rust epidemics and races of Puccinia striiformis f. sp tritici in the United States in 2000. Plant Disease. 86(1):39-46.

Chen X., Penman L., Wan A. and Cheng P. 2010. Virulence races of Puccinia striiformis f. sp tritici in 2006 and 2007 and development of wheat stripe rust and distributions, dynamics, and evolutionary relationships of races from 2000 to 2007 in the United States. Canadian Journal of Plant Pathology-Revue Canadienne De Phytopathologie. 32(3):315-333.

Duan X., Tellier A., Wan A., Leconte M., De V.-P.C. and Enjalbert J. 2010. Puccinia striiformis f.sp. tritici presents high diversity and recombination in the over-summering zone of Gansu, China. Mycologia. 
102(1):44-53.

Enjalbert J., Duan X., Leconte M., Hovmoller M.S. and De Vallavieille-Pope C. 2005. Genetic evidence of local adaptation of wheat yellow rust (Puccinia striiformis f. sp. tritici) within France. Molecular Ecology. 14(7):2065-2073.

Excoffier L., Smouse P.E. and Quattro J.M. 1992. Analysis of molecular variance inferred from metric distances among DNA haplotypes: application to human mitochondrial DNA restriction data. Genetics. 131(2):479-491.

Goss E.M., Tabima J.F., Cooke D.E.L., Restrepo S., Fry W.E., Forbes G.A., Fieland V.J., Cardenas M. and Grunwald N.J. 2014. The irish potato famine pathogen phytophthora infestans originated in central Mexico rather than the Andes. Proceedings of the National Academy of Sciences of the United States of America. 111(24):8791-8796.

Grunwald N.J., Everhart S.E., Knaus B.J. and Kamvar Z.N. 2017. Best practices for population genetic analyses. Phytopathology. 107(9):1000-1010.

Grunwald N.J., Goodwin S.B., Milgroom M.G. and Fry W.E. 2003. Analysis of genotypic diversity data for populations of microorganisms. Phytopathology. 93(6):738-746.

He Z., Zhuang Q., Cheng S., Yu Z., Zhao Z. and Liu X. 2018. Wheat production and technology improvement in China. Journal of Agriculture (in Chinese). 8(1):99-106.

Holtz M.D., Kumar K., Zantinge J.L. and Xi K. 2014. Genetic diversity ofPuccinia striiformis from cereals in Alberta, Canada. Plant Pathology. 63(2):415-424.

Hovmoller M.S., Sorensen C.K., Walter S. and Justesen A.F., 2011. Diversity of Puccinia striiformis on cereals and grasses. In: NK Vanalfen, G Bruening and JE Leach, eds. Annual Review of Phytopathology, Vol 49. Palo Alto: Annual Reviews, 197-217. (Annual Review of Phytopathology; vol. 49.)

Huang C., Jiang Y., Ji G., Zhang G., Li H. and Li Y. 2018a. Spatiotemporal dynamics of wheat stripe rust epidemics at regional level in China in 2017. Journal of Plant Protection (in Chinese). 45(1):20-26.

Huang C., Jiang Y., Li P., Peng H., Cui Y., Yang J. and Xie F. 2018b. Epidemics analysis of wheat stripe rust in China in 2017. Plant protection (in Chinese). 44(2):162-166.

Huang L., Xiao X., Liu B., Gao L., Gong G., Chen W., Zhang M. and Liu T. 2020. Identification of stripe rust resistance genes in common wheat cultivars from the Huang-Huai-Hai region of China. Plant Disease. 104(6):1763-1770.

Jin Y., Szabo L.J. and Carson M. 2010. Century-old mystery of Puccinia striiformis life history solved with the identification of Berberis as an alternate host. Phytopathology. 100(5):432-435.

Kamvar Z.N., Tabima J.F. and Grunwald N.J. 2014. Poppr: an R package for genetic analysis of populations with clonal, partially clonal, and/or sexual reproduction. PeerJ. 2:e281.

Liang J., Liu X., Li Y., Wan Q., Ma Z. and Luo Y. 2016. Population genetic structure and the migration of Puccinia striiformis f. sp. tritici between the Gansu and Sichuan basin populations of China. Phytopathology. 106(2):192-201.

Liang J., Wan Q., Luo Y. and Ma Z. 2013. Population genetic structures of Puccinia striiformis in Ningxia and Gansu provinces of China. Plant Disease. 97(4):501-509.

Liu X., Yuan W., Liang D., Shi X. and Ma Z. 2016. Population genetic structures of Puccinia striiformis f. sp. tritici in Yunnan and Guizhou province. Journal of Yunnan Agricultural University (Natural Science) (in Chinses). 31(5):779-784. 
Luijckx P., Ho E.K.H., Gasim M., Chen S., Stanic A., Yanchus C., Kim Y.S. and Agrawal A.F. 2017. Higher rates of sex evolve during adaptation to more complex environments. Proceedings of the National Academy of Sciences of the United States of America. 114(3):534-539.

Mboup M., Leconte M., Gautier A., Wan A.M., Chen W., De V.C. and Enjalbert J. 2009. Evidence of genetic recombination in wheat yellow rust populations of a Chinese oversummering area. Fungal Genetics and Biology. 46(4):299-307.

Meirmans P.G. and Hedrick P.W. 2011. Assessing population structure: F(ST) and related measures. Molecular Ecology Resources. 11(1):5-18.

Milus E.A., Kristensen K. and Hovmoller M.S. 2009. Evidence for increased aggressiveness in a recent widespread strain of Puccinia striiformis f. sp. tritici causing stripe rust of wheat. Phytopathology. 99(1):89-94.

Nei M. 1972. Genetic distance between populations. American Naturalist. 106(949):283-92.

Nei M. 1978. Estimation of average heterozygosity and genetic distance from a small number of individuals. Genetics. 89(3):583-590.

Peakall R. and Smouse P.E. 2012. GenAlEx 6.5: genetic analysis in excel. population genetic software for teaching and research-an update. Bioinformatics (Oxford, England). 28(19):2537-2539.

Schaal B.A. 1980. Measurement of gene flow in Lupinus texensis . Nature. 284(5755):450-451.

Simpson E.H. 1972. Measurement of diversity. Journal of Cardiothoracic and Vascular Anesthesia. 27(2):261.

Song Q.J., Shi J.R., Singh S., Fickus E.W., Costa J.M., Lewis J., Gill B.S., Ward R. and Cregan P.B. 2005. Development and mapping of microsatellite (SSR) markers in wheat. Theoretical and applied genetics. 110(3):550-560.

Stubbs R.W. 1988. Pathogenicity analysis of yellow (stripe) rust of wheat and its significance in a global context. Chapter 3.

Tian Y., Zhan G., Chen X., Tungruentragoon A., Lu X., Zhao J., Huang L. and Kang Z. 2016. Virulence and simple sequence repeat marker segregation in a Puccinia striiformis $\mathrm{f}$. sp. triticipopulation produced by selfing a Chinese isolate on Berberisshensiana. Phytopathology. 106(2):185-191.

Tibayrenc M., Kjellberg F., Arnaud J., Oury B., Breniere F.S., Darde M.-L. and Ayala F.J. 1991. Are eukaryotic microorganisms clonal or sexual? A population genetics vantage. Proceedings of the National Academy of Sciences of the United States of America. 88(12):5129-5133.

Wan A., Chen X. and He Z. 2007. Wheat stripe rust in China. Australian Journal of Agricultural Research. 58(6):605-619.

Wan Q., Liang J., Luo Y. and Ma Z. 2015. Population Genetic Structure ofPuccinia striiformis in northwestern China. Plant Disease. 99(12):1764-1774.

Wang H., Yang X. and Ma Z. 2010. Long-distance spore transport of wheat stripe rust pathogen from Sichuan, Yunnan, and Guizhou in southwestern China. Plant Disease. 94(7):873-880.

Wang Z., Zhao J., Chen X., Peng Y., Ji J., Zhao S., Lv Y., Huang L. and Kang Z. 2016. Virulence variations of Puccinia striiformis f. sp.tritici isolates collected from Berberis spp. in China. Plant Disease. 100(1):131-138.

Xie S., Wang K., Chen Y. and Chen W. 1993. Preliminary studies on the relationship between transport of wheat stripe rust and the upper air current in China. Acta phytopathologica sinica (in Chinese). 23(3):203209. 
Zeng S. and Luo Y. 2006. Long-distance spread and interregional epidemics of wheat stripe rust in China. Plant Disease. 90(8):980-988.

Zhan G., Wang F., Wan C., Han Q., Huang L., Kang Z. and Chen X. 2016. Virulence and molecular diversity of the Puccinia striiformis f. sp. tritici population in Xinjiang in relation to other regions of western China. Plant Disease. 100(1):99-107.

Zhan G., Zhuang H., Wang F., Wei G., Huang L. and Kang Z. 2013. Population genetic diversity of Puccinia striiformis f. sptritici on different wheat varieties in Tianshui, Gansu province. World Journal of Microbiology and Biotechnology. 29(1):173-181.

Zhang J., Liu B., Chen W., Liu T. and Gao L. 2013. Temperature sensitivity of population of Puccinia striiformis Westend. Acta phytopathologica sinica (in Chinese). 43(1):88-90.

Zhao J., Zheng D., Zuo S., Wang L., Huang L. and Kang Z. 2018. Research advances in alternate host and sexual reproduction of wheat yellow rust pathogen Puccinia striiformis f. sp. tritici Erikss. et Henn. Journal of Plant Protection (in Chinese). 45(1):7-19.

\section{Data Accessibility}

\section{Authorship Contribution}

Heng Yang, Liang Huang, and Aolin Wang carried out the experiment. Liang Huang and Heng Yang analysed the data and wrote the manuscript with support from Taiguo Liu and Chongjing Xia. Taiguo Liu, Wanquan Chen and Xiaohui Kang conceived the original idea. Taiguo Liu, Yilin Zhou, Min Zhang and Wanquan Chen supervised the project. All authors discussed the results and contributed to the final manuscript.

\section{Data Availability Statement}

The data used to support the findings of this study are available from the corresponding author (Taiguo Liu) upon request. We can uploaded all data to a public database when you received the manuscript.

\section{Hosted file}

Tables .pdf available at https://authorea.com/users/373525/articles/491212-molecular-evidencefor-a-new-dispersal-route-of-inoculum-source-for-puccinia-striiformis-f-sp-tritici-incentral-and-eastern-china

\section{Hosted file}

Figures.pdf available at https://authorea.com/users/373525/articles/491212-molecularevidence-for-a-new-dispersal-route-of-inoculum-source-for-puccinia-striiformis-f-sptritici-in-central-and-eastern-china 